\title{
Lattice supersymmetry in 1D with two supercharges
}

\author{
Sergio Arianos*, Alessandro D’Adda ${ }^{\dagger}$ \\ INFN, Torino \\ E-mail: arianoseto.infn.it, daddaeto.infn.it
}

Noboru Kawamoto, Jun Saito

Department of Physics, Hokkaido University

E-mail: kawamoto@particle.sci.hokudai.ac.jp,

saito@particle.sci.hokudai.ac.jp

\begin{abstract}
A consistent formulation of a fully supersymmetric theory on the lattice has been a long standing challenge. In recent years there has been a renewed interest on this problem with different approaches. At the basis of the formulation we present in the following there is the Dirac-Kähler twisting procedure, which was proposed in the continuum for a number of theories, including $N=4$ SUSY in four dimensions. Following the formalism developed in recent papers, an exact supersymmetric theory with two supercharges on a one dimensional lattice is realized using a matrix-based model. The matrix structure is obtained from the shift and clock matrices used in two dimensional non-commutative field theories. The matrix structure reproduces on a one dimensional lattice the expected modified Leibniz rule. Recent claims of inconsistency of the formalism are discussed and shown not to be relevant.
\end{abstract}

The XXV International Symposium on Lattice Field Theory

July 30 - August 42007

Regensburg, Germany

\footnotetext{
* Speaker.

$\dagger$ Speaker.
} 


\section{Introduction}

A consistent formulation of supersymmetry on the lattice is a long standing problem. A number of approaches that allow to preserve exactly one supersymmetry on the lattice in theories with an extended supersymmetry have been proposed in recent years $[1,2,3]$. A more ambitious approach, aiming to preserve exactly all supersymmetries in some extended supersymmetric model, was also proposed $[4,5,6]$.

Like for many of the previous formulations one of the key ingredients of this approach is the use of Dirac-Kähler fermions on the lattice to overcome the doubling problem. Its main feature however is the use of an extended lattice where the standard links, corresponding to the discrete elementary translations on the lattice, are implemented by "fermionic" links that correspond to the action of supersymmetry charges. The need for these extra links was the result of a careful analysis of the modified "shifted" Leibniz rules, that both translations and supersymmetry transformation have for consistency to satisfy on a lattice when acting on a product of (super)fields. The structure of the extended lattice thus reflects the structure of the supersymmetry algebra, and a consistent solution is found only for some specific extended superalgebra like the $N=2$ superalgebra for $D=2[4,5]$, the $N=4$ superalgebra for $D=3[6]$, and the $N=4$ superalgebra for $D=4$ [5]. Consistently the supersymmetry charges are in this approach associated to links, rather than to sites. In connection with this point a number of criticisms were put forward [7, 8], with the claim that the link nature of supercharges leads to inconsistencies and ambiguities in the definition of the supersymmetry transformations. Very recently [9] it was shown that this approach fits within the scheme of Kaplan's orbifold formulation [1] and the actual invariance of the action proposed in [5] under all Susy charges has been questioned.

In order to investigate the above issues we considered a simple one dimensional supersymmetric model with two supercharges, the same model already considered by Bruckmann and de Kok in [7]. A detailed analysis of this model on the lattice will be the subject of a future publication [10] and some of the main results are anticipated in the second part of the present report. In particular it is shown that supersymmetry transformations are consistently and unambiguously determined on the lattice by using the superfield formalism. In the first part of the report we discuss some general features of (one dimensional) lattice theories (not necessarily supersymmetric). In particular we shall study the connection between the modified Leibniz rule mentioned above and the translational invariance of the action on the lattice and show how they follow from dimensional reduction of a two dimensional theory formulated on a non-commutative lattice.

\section{One dimensional lattice models}

\subsection{Matrix representation and modified Leibniz rules}

Consider a one dimensional lattice with $N$ sites and periodic boundary conditions and a scalar field $\varphi$, defined on the sites of the lattice. Let $\varphi_{r}(r=1,2, \cdots, N)$ be the value of the field $\varphi$ on the $r$ th site of the lattice. The $N$ numbers $\varphi_{r}$ can be regarded as the eigenvalues of an $N \times N$ diagonal 
matrix $\varphi$ :

$$
\varphi=\left(\begin{array}{cccccc}
\varphi_{1} & 0 & 0 & 0 & \cdots & 0 \\
0 & \varphi_{2} & 0 & 0 & \cdots & 0 \\
0 & 0 & \varphi_{3} & 0 & \cdots & 0 \\
\vdots & \vdots & \vdots & \vdots & \ddots & \vdots \\
0 & 0 & 0 & 0 & \cdots & \varphi_{N}
\end{array}\right)
$$

whose rows and columns are in one to one correspondence with the sites of the lattice. Notice that the ordering of the rows and columns is the same as the one of the lattice sites, so that neighboring eigenvalues correspond to the values of the field in neighboring sites. Derivatives are replaced on the lattice by finite differences:

$$
\left(\Delta_{+} \varphi\right)_{r}=\varphi_{r+1}-\varphi_{r}
$$

The breaking of the translational invariance due to the discrete nature of the lattice results into a violation of the Leibniz rule when the finite difference of a product of two functions is considered. As discussed in detail in ref. [4], to which we refer for a more exhaustive treatment, a modified Leibniz rule holds in place of the usual one:

$$
\left(\Delta_{+} \varphi \psi\right)_{r}=\left(\Delta_{+} \varphi\right)_{r} \psi_{r}+\varphi_{r+1}\left(\Delta_{+} \psi\right)_{r} .
$$

In matrix notation finite differences may be represented using the shift matrices $\Delta_{+}$and $\Delta_{-}$:

$$
\Delta_{+}=\left(\begin{array}{cccccc}
0 & 1 & 0 & 0 & \cdots & 0 \\
0 & 0 & 1 & 0 & \cdots & 0 \\
0 & 0 & 0 & 1 & \cdots & 0 \\
\vdots & \vdots & \vdots & \vdots & \ddots & \vdots \\
0 & 0 & 0 & 0 & \cdots & 1 \\
1 & 0 & 0 & 0 & \cdots & 0
\end{array}\right), \quad \Delta_{-}=\Delta_{+}^{-1}=\left(\begin{array}{cccccc}
0 & 0 & 0 & \cdots & 0 & 1 \\
1 & 0 & 0 & \cdots & 0 & 0 \\
0 & 1 & 0 & \cdots & 0 & 0 \\
0 & 0 & 1 & \cdots & 0 & 0 \\
\vdots & \vdots & \vdots & \ddots & \vdots & \vdots \\
0 & 0 & 0 & \cdots & 1 & 0
\end{array}\right)
$$

namely, in components

$$
\left(\Delta_{+}\right)_{r s}=\delta_{r, s-1}, \quad\left(\Delta_{-}\right)_{r s}=\delta_{r, s+1} .
$$

In the continuum the derivative $\partial \varphi$ is just the commutator $[\partial, \varphi]$; on the lattice however the commutator $\left[\Delta_{+}, \varphi\right]$ is not diagonal, its non vanishing matrix elements being on a shifted diagonal as in $\Delta_{+}$. In order to write the finite difference (2.2) as a function defined on the lattice sites, namely a diagonal matrix, we have to define it as:

$$
\left(\Delta_{+} \varphi\right)=-\Delta_{+}\left[\Delta_{-}, \varphi\right]=\left(\begin{array}{cccccc}
\varphi_{2}-\varphi_{1} & 0 & 0 & 0 & \cdots & 0 \\
0 & \varphi_{3}-\varphi_{2} & 0 & 0 & \cdots & 0 \\
0 & 0 & \varphi_{4}-\varphi_{3} & 0 & \cdots & 0 \\
\vdots & \vdots & \vdots & \vdots & \ddots & \vdots \\
0 & 0 & 0 & 0 & \cdots & \varphi_{1}-\varphi_{N}
\end{array}\right)
$$

The factor $\Delta_{+}$in front of the commutator is responsible for the violation of the Leibniz rule, in fact we have:

$$
\left(\Delta_{+} \varphi \psi\right)=\left(\Delta_{+} \varphi\right) \psi+\Delta_{+} \varphi \Delta_{-}\left(\Delta_{+} \psi\right)
$$


which is completely equivalent to (2.3). Notice that $\Delta_{+} \varphi \Delta_{-}$is a "shifted" field, where the eigenvalue $\varphi_{r}$ has been replaced by $\varphi_{r+1}:\left(\Delta_{+} \varphi \Delta_{-}\right)_{r}=\varphi_{r+1}$. The modified Leibniz rule (2.7) reflects the fact that translational symmetry on the lattice is a discrete, and not a continuous symmetry. To make this point clear consider an action given as the trace of a product of fields $\varphi_{i}$ :

$$
S=\operatorname{Tr} \varphi_{1} \varphi_{2} \cdots \varphi_{r}
$$

The trace corresponds to the sum over all lattice sites, and translational invariance can be simply expressed as the invariance of (2.8) under

$$
\varphi_{i} \rightarrow \Delta_{+} \varphi_{i} \Delta_{-}=\varphi_{i}+\delta \varphi_{i}
$$

where $\delta \varphi_{i}=\left(\Delta_{+} \varphi\right)$ as defined in (2.6). When the r.h.s. of (2.9) is inserted into (2.8) all orders of $\delta \varphi_{i}$ must be kept in order to preserve the exact symmetry and the variation of the Lagrangian can be cast in the form:

$$
\begin{aligned}
& \delta\left(\varphi_{1} \varphi_{2} \cdots \varphi_{r}\right)=\left(\delta \varphi_{1}\right) \varphi_{2} \cdots \varphi_{r-1} \varphi_{r}+\left(\varphi_{1}+\delta \varphi_{1}\right)\left(\delta \varphi_{2}\right) \varphi_{3} \cdots \varphi_{r}+\cdots \\
& +\left(\varphi_{1}+\delta \varphi_{1}\right)\left(\varphi_{2}+\delta \varphi_{2}\right) \cdots\left(\varphi_{r-1}+\delta \varphi_{r-1}\right)\left(\delta \varphi_{r}\right)
\end{aligned}
$$

which is again the modified Leibniz rule. It is clear that eq. (2.10) follows directly from the transformation (2.9) by keeping all orders in $\delta \varphi_{i}$ while linear terms in $\delta \varphi_{i}$ give the ordinary Leibniz rule typical of the continuum limit. We stressed this point because the situation is different in the supersymmetric theory discussed in the following section: supersymmetry charges are non diagonal and hence supersymmetry transformations of a product of superfields obey a modified Leibniz rule on the lattice, as discussed in section 4 as well as in previous papers [4], however these modified Leibniz rules cannot be derived, at least in the present formulation, from a field transformation as in (2.9).

\subsection{Dimensional reduction and non-commutative lattice}

In the matrix representation of fields (2.1) there is a one-to-one correspondence between rows (or columns) and lattice points. If the lattice contains $N$ points, a generic matrix will have $N^{2}$ matrix elements and each matrix element $\varphi_{i j}$ is associated to an ordered link joining two arbitrary points $i$ and $j$ of the lattice. A generic matrix then describes a completely non-local object on the lattice. In order to recover the lattice structure restriction must be imposed on the matrix $\varphi$ for it to describe a local or almost local field.

Such restrictions are just the analogue, in this simple one dimensional model, of the orbifold conditions used by Kaplan and collaborators in their approach to lattice supersymmetry [1]. In fact they can be expressed, as in [1], in terms of the "clock" matrix $\Omega$ defined by ${ }^{1}$ :

$$
\Omega=\left(\begin{array}{ccccc}
1 & 0 & 0 & \cdots & 0 \\
0 & \omega & 0 & \cdots & 0 \\
0 & 0 & \omega^{2} & \cdots & 0 \\
\vdots & \vdots & \vdots & \ddots & \vdots \\
0 & 0 & 0 & \cdots & \omega^{N-1}
\end{array}\right), \quad \begin{gathered}
\\
\omega^{N}=e^{\frac{-i 2 \pi}{N}}
\end{gathered}
$$

\footnotetext{
${ }^{1}$ In the definition of $\omega$ we adopt the same notation used in [12].
} 
A field defined on the lattice sites and described by a diagonal matrix $\varphi$ simply commutes with $\Omega$, but in general we shall be interested in fields defined on the lattice links (like gauge fields) whose matrix representation is of the form

$$
\varphi_{i j}^{(a)}=\delta_{i+a, j} \varphi_{i}^{(a)}
$$

where $a= \pm 1$ for the link variables while higher values of $a$ denote more non-local fields, like the ones involving higher derivatives. It is easy to check that requiring that the field $\varphi^{(a)}$ is of the form (2.12) is the same as imposing the following orbifold condition:

$$
\varphi^{(a)} \Omega=\omega^{a} \Omega \varphi^{(a)} .
$$

In particular, as the finite difference operator $\Delta_{+}$and its conjugate $\Delta_{-}$are such matrices with $a=1$ and $a=-1$ respectively, we also have:

$$
\Delta_{ \pm} \Omega=\omega^{ \pm 1} \Omega \Delta_{ \pm}
$$

The orbifold conditions (2.13) have an interesting interpretation in terms of non-commutative geometry. Non-commutative geometry on a discrete periodic lattice has been studied by several authors [11, 12]. We adopt the approach of Bars and Minic which is the most convenient for our purpose. The first thing to notice is that before the orbifold conditions are applied a field $\varphi$ is represented by an $N \times N$ matrix, namely it contains $N^{2}$ degrees of freedom, that is exactly the number of degrees of freedom of a scalar field on a two dimensional lattice with $N$ links in each direction. So the reduction of the degrees of freedom from $N^{2}$ to $N$ amounts effectively to some kind of dimensional reduction. In order to understand what type of dimensional reduction that is, let us consider a non-commutative two dimensional lattice as defined in ref. [12]. The lattice structure is imposed by requiring that the two coordinates $X_{1}$ and $X_{2}$ are operators with $N$ discrete eigenvalues $\left(x_{i}\right)_{n}=n a$ where $a$ is the lattice spacing and $n$ an integer modulo $N$. The non-commutativity of the coordinates $X_{1}$ and $X_{2}$ is given by:

$$
\left[\frac{X_{1}}{L}, \frac{X_{2}}{L}\right]=\frac{i}{2 \pi N b}
$$

where $b$ is an arbitrary integer and $L=N a$ is in each direction the size of the "box" with periodic boundary conditions. The quantisation of $b$, as explained in [12], is a consequence of the periodic boundary conditions and of the discreteness of the spectrum of eigenvalues. The normalization of the coordinates in (2.15) has been chosen to show that if the continuum limit is done keeping the size $L$ of the box, namely the infrared cutoff, fixed the r.h.s. vanishes in that limit, as continuum limit and large $N$ limit coincide in this case. We shall choose here for convenience $b=1$. It is shown in [12] that the shift operator $\Delta_{+}$and the clock operator $\Omega$ are the translation operators of one lattice unit in the two lattice directions. In the base where $X_{1}$ is diagonal the shift operator $\Delta_{+}$ is translation operator of one lattice unit along the positive $X_{1}$ direction ( and its conjugate $\Delta_{-}$in the negative direction); $\Omega$ on the other hand is the translation operator of one lattice unit along $X_{2}$. For $b=1$ the translation operators can be written in terms of the coordinate operators as:

$$
\Delta_{+}=\exp i \frac{2 \pi}{N} \frac{X_{2}}{a}=\omega^{-\hat{X}_{2}}, \quad \Omega=\exp -i \frac{2 \pi}{N} \frac{X_{1}}{a}=\omega^{\hat{X}_{1}}
$$


where $\hat{X}_{i}=\frac{X_{i}}{a}$ are the coordinates normalized to the lattice spacing (the eigenvalues of $\hat{X}_{i}$ are integers) and $\omega$ the $N$ th root of the identity, as defined above. The orbifold conditions (2.13) have now a clear interpretation as they represent different types of compactification along the $X_{2}$ direction. In the simplest case of a scalar field the condition $[\varphi, \Omega]=0$ simply states that there is no dependence on the $X_{2}$ direction. However the non-commutativity (2.14) of $\Omega$ and $\Delta_{+}$implies that $\varphi$ and $\left[\Delta_{+}, \varphi\right]$ do not obey the same orbifold/dimensional-reduction conditions, hence the impossibility of defining the finite difference operation as a commutator and the need for a modified Leibniz rule. A more precise understanding of this is obtained, always following ref. [12], by introducing the analogue on a two dimensional non-commutative lattice of the Moyal product. Let us introduce with [12] the most general translation operator:

$$
\hat{v}_{p}=\exp i p_{\mu} X_{\mu}
$$

with $p_{\mu}$ the discretized momenta on the lattice:

$$
p_{1}=-\frac{2 \pi k_{2}}{a N}, \quad p_{2}=\frac{2 \pi k_{1}}{a N}, \quad k_{1}, k_{2} \text { integers. }
$$

By using the relations (2.16) and the Baker-Hausdorff formula $\hat{v}_{p}$ can be written as:

$$
\hat{v}_{p} \equiv \hat{v}_{k_{1}, k_{2}}=\omega^{\frac{k_{1} k_{2}}{2}} \Omega^{k_{2}} \Delta_{+}{ }^{k_{1}}
$$

Notice that because of the Baker-Hausdorff term $\omega^{\frac{k_{1} k_{2}}{2}} \hat{v}_{k_{1}, k_{2}}$ is periodic with period $2 N$ (and not $N$ ) in $k_{1}$ and $k_{2}$, and hence we shall take $k_{1}$ and $k_{2}$ to be integers modulo $2 N$. Given a scalar field $\varphi$ represented by an $N \times N$ matrix $\varphi$ we can define its representation in the space of the discrete momenta $k_{1}$ and $k_{2}$ as:

$$
\hat{\varphi}_{k_{1}, k_{2}}=\frac{1}{N} \operatorname{Tr} \hat{v}_{k_{1}, k_{2}} \varphi
$$

where, due to the $\omega^{\frac{k_{1} k_{2}}{2}}$ factor in (2.19), $k_{1}$ and $k_{2}$ are defined modulo $2 N$ with the symmetry:

$$
\hat{\varphi}_{k_{1}+N, k_{2}}=(-1)^{k_{2}} \hat{\varphi}_{k_{1}, k_{2}} \quad \text { and } \quad \hat{\varphi}_{k_{1}, k_{2}+N}=(-1)^{k_{1}} \hat{\varphi}_{k_{1}, k_{2}} .
$$

The coordinate representation of $\varphi$ can be obtained by doing a discrete Fourier transform of $\hat{\varphi}_{k_{1}, k_{2}}$ :

$$
\varphi(\xi)=\frac{1}{N} \sum_{k_{1}, k_{2}} \omega^{k_{1} \hat{\xi}_{2}-k_{2} \hat{\xi}_{1}} \hat{\varphi}_{k_{1}, k_{2}}=\frac{1}{N} \operatorname{Tr} \hat{\Delta}(\xi) \varphi
$$

where we have introduced the matrix $\hat{\Delta}(\xi)$ defined as:

$$
\hat{\Delta}(\xi)=\frac{1}{N} \sum_{p} \operatorname{expip} \mu\left(X_{\mu}-\xi_{\mu}\right)=\frac{1}{N} \sum_{k_{1}, k_{2}} \omega^{\frac{k_{1} k_{2}}{2}} \Omega^{k_{2}} \Delta_{+}{ }^{k_{1}} \omega^{k_{1} \hat{\xi}_{2}-k_{2} \hat{\xi}_{1}} .
$$

As before $\hat{\xi}_{i}=\frac{\xi_{i}}{a}$ are normalized to the lattice spacing and the sum over $k_{i}$ goes from 1 to $2 N$. With $k_{1}$ and $k_{2}$ defined modulo $2 N$ the lattice positions $\hat{\xi}_{i}$ can take both integer and half-integer values (modulo $N$ ), with the integer values coming (thanks to the symmetry (2.21)) from the even values of $k_{i}$ and the half-integer values from the odd values of $k_{i}$. The matrix $\hat{\Delta}(\xi)$ provides through eq. (2.22) 
the map between the matrix representation of the field $\varphi$ and its lattice coordinate representation. Eq. (2.22) can be inverted:

$$
\varphi=\sum_{\xi_{\mu}} \varphi(\xi) \hat{\Delta}(\xi)
$$

The non-commutativity of the coordinates has as a consequence that the product of fields is not local in the coordinate representation: it is the lattice analogue of the Moyal product. It is defined, as in [12], by the relation:

$$
\varphi_{1} \diamond \varphi_{2}(\xi)=N^{-1} \operatorname{Tr} \varphi_{1} \varphi_{2} \hat{\Delta}(\xi)=N^{-1} \sum_{\hat{x}, \hat{y}} \omega^{-2 \varepsilon^{\mu v}\left(\hat{x}_{\mu}-\hat{\xi}_{\mu}\right)\left(\hat{y}_{v}-\hat{\xi}_{v}\right)} \varphi_{1}(\hat{x}) \varphi_{2}(\hat{y})
$$

and denoted as "diamond" product. Further properties of the diamond product can be found in [12], our aim here is to study what it becomes when the orbifold (dimensional reduction) conditions (2.13) are imposed. Let us then consider a field $\varphi^{(a)}$ of the shifted diagonal form (2.12), namely satisfying the orbifold condition (2.13). By using the explicit expression of $\hat{\Delta}(\xi)$ given in (2.23) one can easily find the coordinate representation of the field $\varphi^{(a)}$ :

$$
\varphi^{(a)}(\xi)=\frac{1}{N} \omega^{-a \hat{\xi}_{2}} \varphi_{\hat{\xi}_{1}+\frac{a}{2}-1}^{(a)}
$$

As a result of the dimensional reduction the dependence on $\hat{\xi}_{2}$ in (2.26) is trivial, with the shift $a$ interpreted as a constant momentum in the compactified direction $\xi_{2}$. The index $\hat{\xi}_{1}-\frac{a}{2}$ in (2.26) should be an integer, so fields with even shifts $a$ are defined on integers values of $\hat{\xi}_{1}$ while fields with odd shift $a$ are defined on half-integer values of $\hat{\xi}_{1}$. Consider now two shifted diagonal fields $\varphi_{1}^{\left(a_{1}\right)}$ and $\varphi_{2}^{\left(a_{2}\right)}$. In the matrix representation the product of the two fields is a shifted diagonal matrix with shift equal to $a_{1}+a_{2}$. In the coordinate representation the diamond product of the two fields can be easily calculated and is given by:

$$
\varphi_{1}^{\left(a_{1}\right)} \diamond \varphi_{2}^{\left(a_{2}\right)}(\xi)=N \varphi_{1}^{\left(a_{1}\right)}\left(\hat{\xi}_{1}-\frac{a_{2}}{2}, \hat{\xi}_{2}\right) \varphi_{2}^{\left(a_{2}\right)}\left(\hat{\xi}_{2}+\frac{a_{1}}{2}, \hat{\xi}_{2}\right) .
$$

Given the trivial dependence on $\hat{\xi}_{2}$ of both terms, the $\hat{\xi}_{2}$ dependence of the r.h.s. is just $\omega^{-\left(a_{1}+a_{2}\right)} \hat{\xi}_{2}$, namely the product field has a shift (i.e. momentum in $\xi_{2}$ direction) equal to $a_{1}+a_{2}$. Eq. (2.27) shows that the diamond product is non-commutative also the dimensional reduction conditions have been imposed. This is due to the shifts in the $\hat{\xi}$ dependence at the r.h.s. of (2.27). This type of "mild" non-commutativity is just the one introduced in [4] in order to have all supersymmetries exactly preserved on the lattice. It can also be interpreted in terms of link variables according to the scheme developed in [5] for supersymmetric lattice gauge theories. In fact if we consider $\varphi^{(a)}\left(\hat{\xi}_{1}, \hat{\xi}_{2}\right)$ as a degree of freedom associated in the $\xi_{1}$ space to the link of length $a\left(\hat{\xi}_{1}-\frac{a}{2}, \hat{\xi}_{1}+\frac{a}{2}\right)$, the diamond product (2.27) can be interpreted as the product of two successive link variable of length $a_{1}$ and $a_{2}$ starting in $\hat{\xi}_{1}-\frac{a_{1}+a_{2}}{2}$ and ending in $\hat{\xi}_{1}+\frac{a_{1}+a_{2}}{2}$. 


\section{The $N=2$ supersymmetric model in one dimension}

\subsection{Matrix representation of a Grassmann algebra}

In order to define the $N=2$ supersymmetric quantum mechanics on a one dimensional lattice it is convenient to introduce a matrix representation for the two Grassmann variables $\theta_{1}$ and $\theta_{2}$

$$
\begin{aligned}
\theta_{1} & \equiv \sigma_{+} \otimes \mathbf{1} \otimes \Delta_{+}, & \theta_{2} & \equiv \sigma_{3} \otimes \sigma_{+} \otimes \Delta_{-}, \\
\frac{\partial}{\partial \theta_{1}} & \equiv \sigma_{-} \otimes \mathbf{1} \otimes \Delta_{-}, & \frac{\partial}{\partial \theta_{2}} & \equiv \sigma_{3} \otimes \sigma_{-} \otimes \Delta_{+} ;
\end{aligned}
$$

or explicitly

$$
\begin{array}{ccc}
\theta_{1} \equiv\left(\begin{array}{cccc}
0 & 0 & \Delta_{+} & 0 \\
0 & 0 & 0 & \Delta_{+} \\
0 & 0 & 0 & 0 \\
0 & 0 & 0 & 0
\end{array}\right) & \theta_{2} \equiv\left(\begin{array}{cccc}
0 & \Delta_{-} & 0 & 0 \\
0 & 0 & 0 & 0 \\
0 & 0 & 0 & -\Delta_{-} \\
0 & 0 & 0 & 0
\end{array}\right) \\
\frac{\partial}{\partial \theta_{1}} \equiv\left(\begin{array}{cccc}
0 & 0 & 0 & 0 \\
0 & 0 & 0 & 0 \\
\Delta_{-} & 0 & 0 & 0 \\
0 & \Delta_{-} & 0 & 0
\end{array}\right) & \frac{\partial}{\partial \theta_{2}} \equiv\left(\begin{array}{cccc}
0 & 0 & 0 & 0 \\
\Delta_{+} & 0 & 0 & 0 \\
0 & 0 & 0 & 0 \\
0 & 0 & -\Delta_{+} & 0
\end{array}\right)
\end{array}
$$

where the entries of the above matrices are $N \times N$ matrices and $\Delta_{+}$and $\Delta_{-}$are the shift matrices defined in (2.4).

It is straightforward to check that the matrices (3.3) and (3.4) satisfy the standard Grassmann algebra of the $\theta$ variables. This matrix representation is quite general and can be easily extended to an arbitrary number $n$ of variables by using direct products of $n$ Pauli matrices, namely $2^{n} \times 2^{n}$ matrices. Notice also that according to the approach of ref [4] both $\theta_{i}$ and $\partial \theta_{i}$ contain a shift operator $\Delta_{+}$(resp. $\Delta_{-}$) of one lattice unit, implying that ordinary derivative will correspond to a shift of two lattice spacings.

\subsection{Fields and superfields}

The next ingredient we need in order to construct a supersymmetric lattice theory is a matrix representation of the fields. As usual we deal with bosonic fields, fermionic fields and superfields, defined as follows.

- Bosonic field: a field which commutes with all $\theta$ 's and $\frac{\partial}{\partial \theta}$ 's. A straightforward calculation gives

$$
\hat{\varphi} \equiv\left(\begin{array}{cccc}
\varphi & 0 & 0 & 0 \\
0 & \Delta_{+} \varphi \Delta_{-} & 0 & 0 \\
0 & 0 & \Delta_{-} \varphi \Delta_{+} & 0 \\
0 & 0 & 0 & \varphi
\end{array}\right) \quad \begin{aligned}
\varphi & \equiv N \times N \text { matrix } \\
\theta_{i} \hat{\varphi} & =\hat{\varphi} \theta_{i} \\
\frac{\partial}{\partial \theta_{i}} \hat{\varphi} & =\hat{\varphi} \frac{\partial}{\partial \theta_{i}}
\end{aligned}
$$


- Fermionic field: a field which anticommutes with all $\theta$ 's and $\frac{\partial}{\partial \theta}$ 's. A straightforward calculation gives

$$
\hat{\psi} \equiv\left(\begin{array}{cccc}
\psi & 0 & 0 & 0 \\
0 & -\Delta_{+} \psi \Delta_{-} & 0 & 0 \\
0 & 0 & -\Delta_{-} \psi \Delta_{+} & 0 \\
0 & 0 & 0 & \psi
\end{array}\right) \quad \begin{aligned}
\psi & \equiv N \times N \text { fermionic matrix } \\
\theta_{i} \hat{\psi} & =-\hat{\psi} \theta_{i} \\
\frac{\partial}{\partial \theta_{i}} \hat{\psi} & =-\hat{\psi} \frac{\partial}{\partial \theta_{i}}
\end{aligned}
$$

- Superfield: a field which commutes with all $\theta$ 's but not with $\frac{\partial}{\partial \theta}$ 's. It has a standard expansion in powers of $\theta$ 's:

$$
\Phi=\hat{\varphi}+\theta_{1} \hat{\psi}_{1}+\theta_{2} \hat{\psi}_{2}+\theta_{1} \theta_{2} \hat{D} .
$$

In our matrix representation it can be written as

$$
\Phi=\left(\begin{array}{cccc}
\varphi-\psi_{2} \Delta_{-} & -\psi_{1} \Delta_{+} & -D \\
0 & \Delta_{+} \varphi \Delta_{-} & 0 & \Delta_{+} \psi_{1} \\
0 & 0 & \Delta_{-} \varphi \Delta_{+} & -\Delta_{-} \psi_{2} \\
0 & 0 & 0 & \varphi
\end{array}\right)
$$

From equations $(3.5,3.6,3.8)$ it is apparent that the actual building blocks of the model are the matrices "without hat" $\varphi, \psi_{1}, \psi_{2}$ and $D$. Indeed these are the matrices which will be identified with the usual fields in the continuum limit.

So far $\varphi, \psi_{1}, \psi_{2}$ and $D$ are arbitrary $N \times N$ matrices with $N^{2}$ degrees of freedom. In order to describe a one dimensional lattice of size $N$ we need to apply to $\Phi$ an orbifold condition as in (2.13), namely:

$$
[\hat{\Omega}, \Phi]=0,
$$

where $\hat{\Omega}$ is the block diagonal matrix $\hat{\Omega}=\mathbf{1} \otimes \mathbf{1} \otimes \Omega$. This orbifold condition may also be interpreted as a dimensional reduction from a two-dimensional non-commutative lattice, but we will not discuss this possibility here.

In terms of the component fields eq.(2.13) requires:

$$
\begin{array}{r}
{[\Omega, \varphi]=[\Omega, D]=0} \\
\Omega \psi_{1}-\omega \psi_{1} \Omega=0 \\
\Omega \psi_{2}-\omega^{-1} \psi_{2} \Omega=0
\end{array}
$$

This means that $\varphi$ and $D$ are diagonal matrices; $\psi_{1}$ (like $\Delta_{-}$) has non vanishing elements only on the one-down diagonal; $\psi_{2}$ (like $\Delta_{+}$) has non vanishing elements only on the one-up diagonal. Notice however that in the block matrix representation (3.8) all entries are diagonal $N \times N$ matrices.

\subsection{Supercharges and susy transformations}

The two supercharges of the $N=2$ supersymmetric quantum mechanics are given in the continuum theory by

$$
Q_{i}=\frac{\partial}{\partial \theta_{i}}+\theta_{i} \frac{\partial}{\partial t}
$$


As we assigned to $\theta_{i}$ a shift operator corresponding to one lattice unit, the time derivative $\partial t$ will be associated on the lattice to the two units shift operator $\Delta_{ \pm}^{2}$. The correspondence between continuum and lattice operators will then be

$$
\partial t \rightarrow N \Delta_{ \pm}^{2}
$$

where the factor $N$ is needed to recover the continuum limit. In fact $\left[\Delta_{ \pm}^{2}, \varphi\right]$ is of order of the lattice spacing $a=\frac{L}{N}$, namely, if the size $L$ of the lattice is kept fixed, of order $1 / N$. In our lattice formulation, both the $\theta$ 's and $\frac{\partial}{\partial \theta}$ carry a shift. On the other hand it is necessary for consistency that the two terms in $Q_{i}$ carry the same shift, and this determines the supercharges on the lattice without ambiguity:

$$
Q_{1}=\frac{\partial}{\partial \theta_{1}}+N \theta_{1} \hat{\Delta}_{-}^{2}=\left(\begin{array}{cccc}
0 & 0 & N \Delta_{-} & 0 \\
0 & 0 & 0 & N \Delta_{-} \\
\Delta_{-} & 0 & 0 & 0 \\
0 & \Delta_{-} & 0 & 0
\end{array}\right), \quad Q_{2}=\frac{\partial}{\partial \theta_{2}}+N \theta_{2} \hat{\Delta}_{+}^{2}=\left(\begin{array}{cccc}
0 & N \Delta_{+} & 0 & 0 \\
\Delta_{+} & 0 & 0 & 0 \\
0 & 0 & 0 & -N \Delta_{+} \\
0 & 0 & -\Delta_{+} & 0
\end{array}\right)
$$

$Q_{1}$ and $Q_{2}$ defined in (3.13) satisfy the algebra of supersymmetric quantum mechanics written in Majorana representation (see [7] and references therein) namely:

$$
\begin{aligned}
Q_{1}^{2} & =N \hat{\Delta}_{-}^{2}, & Q_{2}^{2} & =N \hat{\Delta}_{+}^{2}, \\
\left\{Q_{1}, Q_{2}\right\} & =0, & {\left[Q_{1,2}, \Delta_{ \pm}\right] } & =0 .
\end{aligned}
$$

Supersymmetry transformations are naively obtained by taking the commutator of $Q_{1}$ and $Q_{2}$ with $\Phi$. However, for consistency we want the supersymmetry variations of $\Phi$ to commute with $\Omega$, just like $\Phi$, and also to commute with all the $\theta$ 's. This is obtained by defining

$$
\delta_{1} \Phi=\hat{\eta}_{1} \hat{\Delta}_{+}\left[Q_{1}, \Phi\right], \quad \delta_{2} \Phi=\hat{\eta}_{2} \hat{\Delta}_{-}\left[Q_{2}, \Phi\right]
$$

where

$$
\hat{\eta}_{1}=\eta_{1}\left(\begin{array}{cccc}
\mathbf{1} & 0 & 0 & 0 \\
0 & -\mathbf{1} & 0 & 0 \\
0 & 0 & -\mathbf{1} & 0 \\
0 & 0 & 0 & \mathbf{1}
\end{array}\right), \quad \hat{\eta}_{2}=\eta_{2}\left(\begin{array}{cccc}
\mathbf{1} & 0 & 0 & 0 \\
0 & -\mathbf{1} & 0 & 0 \\
0 & 0 & -\mathbf{1} & 0 \\
0 & 0 & 0 & \mathbf{1}
\end{array}\right)
$$

and $\eta_{1}, \eta_{2}$ are odd Grassmann parameters:

$$
\eta_{i} \psi_{j}=-\psi_{j} \eta_{i} \quad \forall i, j=1,2 .
$$

The matrix in (3.17) anticommutes with all $\theta$ 's, so that $\hat{\eta}_{i}$ anticommutes with both $\theta$ 's and the fermionic fields.

By doing explicit matrix computations we obtain, in terms of component fields

$$
\begin{aligned}
\delta_{1} \varphi & =\eta_{1} \Delta_{+} \psi_{1}, & \delta_{1} D & =\eta_{1} N \Delta_{+}\left[\Delta_{-}^{2}, \psi_{2}\right], \\
\delta_{1} \psi_{1} & =-\eta_{1} N \Delta_{+}\left[\Delta_{-}^{2}, \varphi\right], & \delta_{1} \psi_{2} & =-\eta_{1} \Delta_{+} D .
\end{aligned}
$$


In the same way, for $\delta_{2}$ we have

$$
\begin{aligned}
\delta_{2} \varphi & =\eta_{2} \Delta_{-} \psi_{2}, & \delta_{2} D & =-\eta_{2} N \Delta_{-}\left[\Delta_{+}^{2}, \psi_{1}\right], \\
\delta_{2} \psi_{1} & =\eta_{2} \Delta_{-} D, & \delta_{2} \psi_{2} & =-\eta_{2} N \Delta_{-}\left[\Delta_{+}^{2}, \varphi\right] .
\end{aligned}
$$

It can be easily verified that these susy transformations form a closed algebra. For instance if

$$
\delta_{2} \Phi=\hat{\eta}_{2} \hat{\Delta}_{-}\left[Q_{2}, \Phi\right], \quad \delta_{2}^{\prime} \Phi=\hat{\eta}_{2}^{\prime} \hat{\Delta}_{-}\left[Q_{2}, \Phi\right],
$$

we have

$$
\left(\delta_{2} \delta_{2}^{\prime}-\delta_{2}^{\prime} \delta_{2}\right) \Phi=2 \eta_{2}^{\prime} \eta_{2} \hat{\Delta}_{-}^{2}\left\{Q_{2},\left[Q_{2} \Phi\right]\right\}=2 \eta_{2}^{\prime} \eta_{2} \hat{\Delta}_{-}^{2}\left[\hat{\Delta}_{+}^{2}, \Phi\right] .
$$

Likewise for $\delta_{1}$ :

$$
\left(\delta_{1} \delta_{1}^{\prime}-\delta_{1}^{\prime} \delta_{1}\right) \Phi=2 \eta_{1}^{\prime} \eta_{1} \hat{\Delta}_{+}^{2}\left[\hat{\Delta}_{-}^{2}, \Phi\right]
$$

and

$$
\left(\delta_{1} \delta_{2}-\delta_{2} \delta_{1}\right) \Phi=0
$$

\section{Why there is no inconsistency}

The supersymmetry variation of a product of two superfields follows a modified Leibniz rule. For instance, if we consider the variation under $Q_{1}$ we have:

$$
\delta_{1}\left(\Phi_{1} \Phi_{2}\right)=\hat{\eta}_{1} \hat{\Delta}_{+}\left[Q_{1}, \Phi_{1} \Phi_{2}\right]=\left(\delta_{1} \Phi_{1}\right) \Phi_{2}+\left(\hat{\Delta}_{+} \Phi_{1} \hat{\Delta}_{-}\right) \delta_{1} \Phi_{2},
$$

where $\hat{\Delta}_{+} \Phi_{1} \hat{\Delta}_{-}$is a shifted field. Similar expression can be obtained for variations under $Q_{2}$ and for translations. Let us denote by $\left.\Phi\right|_{0} \equiv \hat{\varphi}$ the first component of the superfield $\Phi$ in the $\theta$ expansion, namely its diagonal part in the matrix representation of eq. (3.5). As superfields are represented by triangular matrices we have:

$$
\left.\left(\Phi_{1} \Phi_{2}\right)\right|_{0}=\hat{\varphi}_{1} \hat{\varphi}_{2}=\hat{\varphi}_{2} \hat{\varphi}_{1}=\left.\left(\Phi_{2} \Phi_{1}\right)\right|_{0}
$$

Nonetheless, superfields do not commute:

$$
\Phi_{1} \Phi_{2} \neq \Phi_{2} \Phi_{1}
$$

Let us go back to eq. (4.1) and take the diagonal part (i.e. first component) of both terms. We get

$$
\begin{aligned}
& \left.\delta_{1}\left(\Phi_{1} \Phi_{2}\right)\right|_{0}=\delta_{1}\left(\hat{\varphi}_{1} \hat{\varphi}_{2}\right)=\hat{\eta}_{1} \hat{\Delta}_{+}\left(\hat{\psi}_{1}^{(1)} \hat{\varphi}_{2}+\hat{\varphi}_{1} \hat{\psi}_{1}^{(2)}\right), \\
& \left.\delta_{1}\left(\Phi_{2} \Phi_{1}\right)\right|_{0}=\delta_{1}\left(\hat{\varphi}_{2} \hat{\varphi}_{1}\right)=\hat{\eta}_{1} \hat{\Delta}_{+}\left(\hat{\psi}_{1}^{(2)} \hat{\varphi}_{1}+\hat{\varphi}_{2} \hat{\psi}_{1}^{(1)}\right) .
\end{aligned}
$$

In [7] it is claimed that equations (4.4) and (4.5) constitute a contradiction because their r.h.s. are different whereas the 1.h.s. happen to coincide. In fact there is no contradiction. In eq. (4.4) $\hat{\varphi}_{1} \hat{\varphi}_{2}$ is regarded as first component (diagonal part) of $\Phi_{1} \Phi_{2}$, while in eq. (4.5) $\hat{\varphi}_{2} \hat{\varphi}_{1}\left(=\hat{\varphi}_{1} \hat{\varphi}_{2}\right)$ is regarded as first component of $\Phi_{2} \Phi_{1}$. As a matter of fact, in a supersymmetric field theory only transformations of superfields are well defined. Transformations of single components are well defined only if all the components of the superfield are specified. In particular if the action is given in terms of superfields its variation with respect to supersymmetry transformations is unambiguously determined. 


\section{The action}

The usual action of supersymmetric quantum mechanics is given by

$$
S_{\text {cont }}=\int \mathrm{d} x \mathrm{~d} \theta_{1} \mathrm{~d} \theta_{2}\left[\frac{1}{2} \mathscr{D}_{2} \Phi \mathscr{D}_{1} \Phi+\mathrm{i} F(\Phi)\right]
$$

where $F(\Phi)$ is a superpotential. Here our aim is to construct a matrix (lattice) action which reproduces (5.1) in the continuum limit. First of all we need a matrix representation for the covariant derivatives. Following the same reasoning which led to matrix supercharges (3.13) we define

$$
\mathscr{D}_{1}=\frac{\partial}{\partial \theta_{1}}-N \theta_{1} \hat{\Delta}_{-}^{2}, \quad \quad \mathscr{D}_{2}=\frac{\partial}{\partial \theta_{2}}-N \theta_{2} \hat{\Delta}_{+}^{2} .
$$

It is straightforward matrix algebra to check that these covariant derivatives anticommute with all supercharges

$$
\left\{\mathscr{D}_{i}, Q_{j}\right\}=0 \quad \forall i, j \quad \text { and also } \quad\left\{\mathscr{D}_{1}, \mathscr{D}_{2}\right\}=0 .
$$

Besides we have

$$
\mathscr{D}_{1}^{2}=-N \hat{\Delta}_{-}^{2}, \quad \quad \mathscr{D}_{2}^{2}=-N \hat{\Delta}_{+}^{2} .
$$

A suitable candidate for our matrix action is the following

$$
S=\operatorname{Tr}\left(\left\{\frac{\partial}{\partial \theta_{2}},\left[\frac{\partial}{\partial \theta_{1}}, \frac{1}{2}\left[\mathscr{D}_{1}, \Phi\right]\left[\mathscr{D}_{2}, \Phi\right]+\mathrm{i} F(\Phi)\right]\right\}\right)
$$

which for the kinetic reads in terms of the component fields:

$$
S_{k i n} \propto \operatorname{Tr}\left(-N \psi_{1}\left[\Delta_{+}^{2}, \psi_{1}\right]-D^{2}-N^{2}\left[\Delta_{-}^{2}, \varphi\right]\left[\Delta_{+}^{2}, \varphi\right]+N\left[\Delta_{-}^{2}, \psi_{2}\right] \psi_{2}\right) .
$$

The invariance of (5.5) under supersymmetry transformations can be easily proved. First we notice that in (5.5) the derivatives with respect to $\theta_{i}$ can be replaced by the corresponding $Q_{i}$ without affecting the trace. We can write then

$$
S=\operatorname{Tr}\left(\left\{Q_{2},\left[Q_{1}, \frac{1}{2} \Psi_{1} \Psi_{2}+\mathrm{i} F(\Phi)\right]\right\}\right)
$$

where we have defined the fermionic superfields

$$
\Psi_{1}=\left[\mathscr{D}_{1}, \Phi\right], \quad \Psi_{2}=\left[\mathscr{D}_{2}, \Phi\right]
$$

that satisfy the orbifold condition. Let us consider now the variation $\delta_{1} S$ of the action defined according to eq. (3.16):

$$
\delta_{1} S=\operatorname{Tr}\left(\left\{Q_{2},\left[Q_{1}, \hat{\eta}_{1} \hat{\Delta}_{+}\left[Q_{1}, \frac{1}{2} \Psi_{1} \Psi_{2}+\mathrm{i} F(\Phi)\right]\right]\right\}\right) .
$$

By using Jacobi identities and eq.s (3.14) it is easily seen that the expression under trace in (5.9) is a commutator of $\hat{\Delta}^{2}$ with something and hence it vanishes when the trace is taken. The invariance of the action is then proved in complete generality. 


\section{Conclusions}

We have shown, in the simple one dimensional example of $N=2$ supersymmetric quantum mechanics, that supersymmetry transformations on the lattice can be defined without any ambiguity with the aid of the modified Leibniz rule if the superfield formalism is consistently used. We expect that the situation of higher dimensional models will be similar. We already showed in [5] that the $N=2$ supersymmetric Yang-Mills theory in two dimensions can be formulated on the lattice in a way that the action is exact with respect to the four nilpotent supersymmetry charges, thus ensuring exact supersymmetry under all of them. We also showed that $N=4$ supersymmetric Yang-Mills theory in three dimensions can be formulated on the lattice in a similar way [6].

There is however a difference between supersymmetry and, for instance, translational invariance on the lattice. Supersymmetry transformations are defined through eq.s (3.16) and the modified Leibniz rules. However, at least to our understanding, the latter are not the result, as in the case of translations, of a well defined transformation on the fields (or superfields) as in (2.9). This is related to the specific nature of supersymmetry and to the vanishing of powers higher than one of the supersymmetry parameters $\eta_{i}$, which makes it difficult to conceive how the modified Leibniz rules could result from the higher orders in the supersymmetry variation. Some new idea might be necessary to achieve that, and work is in progress towards that aim. The situation may be summarized by saying that we have the exact symmetry, but not the corresponding field transformations. On one hand this suggests that the problem is not fully understood yet, as already mentioned, and on the other hand is not without consequences as it appears problematic to write exact Ward identities without knowing the underlying symmetry transformations of the (super)fields. Work is in progress in this direction too.

\section{References}

[1] D. B. Kaplan, E. Katz and M. Ünsal, JHEP 0305 (2003) 037 [arXiv:hep-lat/0206019]. A. G. Cohen, D. B. Kaplan, E. Katz and M. Ünsal, JHEP 0308 (2003) 024 [arXiv:hep-lat/0302017]; JHEP 0312 (2003) 031 [arXiv:hep-lat/0307012].

[2] S. Catterall and S. Karamov, Phys. Rev. D65 (2002) 094501 [arXiv:hep-lat/0108024]; Phys. Rev. D68 (2003) 014503 [arXiv:hep-lat/0305002].

S. Catterall, JHEP 0305 (2003) 038 [arXiv:hep-lat/0301028].

S. Catterall and S. Ghadab, JHEP 0405 (2004) 044 [arXiv:hep-lat/0311042], JHEP 0610, 063 (2006) [arXiv:hep-lat/0607010]. S. Catterall, JHEP 0411 (2004) 006 [arXiv:hep-lat/0410052]; JHEP 0506, 027 (2005) [arXiv:hep-lat/0503036].

[3] F. Sugino, JHEP 0401 (2004) 015 [arXiv:hep-lat/0311021], JHEP 0403 (2004) 067 [arXiv:hep-lat/0401017], JHEP 0501 (2005) 016 [arXiv:hep-lat/0410035], Phys.Lett. B635 (2006) 218 [arXiv:hep-lat/0601024].

[4] A. D’Adda, I. Kanamori, N. Kawamoto and K. Nagata, Nucl. Phys. B 707 (2005) 100 [arXiv:hep-lat/0406029].

[5] A. D’Adda, I. Kanamori, N. Kawamoto and K. Nagata, Phys. Lett. B 633 (2006) 645 [arXiv:hep-lat/0507029].

[6] A. D’Adda, I. Kanamori, N. Kawamoto and K. Nagata, [arXiv:hep-lat/0707.3533]

[7] F. Bruckmann and M. de Kok, Phys. Rev. D 73 (2006) 074511 [arXiv:hep-lat/0603003]. 
[8] F. Bruckmann, S. Catterall and M. de Kok, [arXiv:hep-lat/0611001].

[9] P. H. Damgaard and S. Matsuura, [arXiv:hep-lat/0708.4129]

[10] S. Arianos, A. D’Adda, N. Kawamoto and J. Saito, to appear.

[11] J. Ambjorn, Y. M. Makeenko, J. Nishimura, R. J. Szabo, JHEP 9911 (1999) 029 [arXiv:hep-th/9911041]; JHEP 0005 (2000) 023 [arXiv:hep-th/0004147].

[12] I. Bars and D. Minic, Phys. Rev. D 62 (2000) 105018 [arXiv:hep-th/9910091]. 